\title{
Increasing motivation and decreasing sensitivity: Dental challenge in eating disorder
}

\author{
Francesca Zotti* \\ Department of Surgery, Dentistry, Paediatrics and Gynecology, University of Verona, Italy
}

Anorexia nervosa (AN) cause dental erosions. This process could be highly damaging both because of increase of dentinal sensitivity, both because of serious dental surface modification. These two conditions make more difficult to approach the patients suffering for AN and to give them support to manage their pathological situation.

\section{Questions}

It is possible to test whether a temporary dental restoration (indirect mockup) could be significant in decreasing dentinal sensitivity and improving psychological motivation in 18 -years old girl affected by AN for five years.

\section{Materials and methods}

Young patient received a questionnaire about dentinal hypersensitivity, dental hygiene habits and frequency of vomiting episodes. Answers showed a high self-reported degree of dentinal sensitivity, with difficult in to eat and drink. Poor level of dental hygiene was noticed (once per day tooth brushing and not flossing) and about three episodes of vomiting in a week.

After stabilization of dental posterior dimension with provisional crowns, a six-teeth temporary restoration in composite resine on upper anterior sector made by CADCAM technology without preparation of teeth surfaces was placed. The mockup was cemented by glass ionomer cement after application of desensitizer (BisBlock. Bisco Inc.). The restoration covered anterior and posterior surfaces of social six sector in order to obtain a good aesthetic result and decrease of sensitivity. The questionnaire was submitted one week after restoration placement.

\section{Results}

Patient reported immediate decrease of dentinal hypersensitivity and a progress in drinking cold beverage. Vomiting episodes did not decrease but dental hygiene have improved (two times per day tooh brushing and once a day flossing). Patient reported in the final questionnaire a psychological upgrading because of possibility in drinking and smiling.

Dentinal sensitivity is of course reduced with immediate coverage of eroded dental surfaces, but we can conclude that a better teeth aesthetic condition might improve also the psychological context in AN.

\section{Conclusions}

It might be positive to introduce temporary restorations in young patients suffering for AN. The aesthetic acceptance and motivation play a positive role in improving health approach. The decrease of hypersensitivity makes easier to eat and drink.

Of course, an adequate psychological and behavioural support is needed in order to correct behaviour and to stabilize achieved results.

The CAD/CAM technology with the feasibility to obtain fast temporary restorations might open a new scenario in rehabilitation of hard cases of erosions and lack of hard tissues, it might be so possible to use a mock-up like a preview of definitive results.

This approach is strongly effective in improving the decrease of hypersensitivity and in empowering the motivation in patients affected by AN.

\section{References}

1. Johansson AK, Omar R, Carlsson GE, Johansson A (2012) Dental erosion and its growing importance in clinical practice: From past to present. Int J Dent 2012: 632907.

2. John P, Muthukumar B, Kumar MV (2015) Comparison of the effect of dentin bonding, dentin sealing agents on the microleakage of provisional crowns fabricated with direct and indirect technique-an invitro study. J Clin Diagn Res 9: ZC54-57. [Crossref]

3. Shetty RM, Bhat S, Mehta D, Srivatsa G, Shetty YB (2012) Comparative analysis of postcementation hypersensitivity with glass ionomer cement and a resin cement: an in vivo study. J Contemp Dent Pract 13: 327-331. [Crossref]

4. Gupta N, Reddy UN, Vasundhar PL, Ramarao KS, Varma KP, Vinod V (2013) Effectiveness of desensitizing agents in relieving the pre- and postcementation sensitivity for full coverage restorations: a clinical evaluation. J Contemp Dent Pract 14: 858-865. [Crossref]
Copyright: (C2017 Zotti F.This is an open-access article distributed under the terms of the Creative Commons Attribution License, which permits unrestricted use, distribution, and reproduction in any medium, provided the original author and source are credited.
Correspondence to: Francesca Zotti, Researcher, Department of Surgery, Dentistry, Paediatrics and Gynecology, University of Verona, Italy, E-mail: francesca.zotti@hotmail.it

Key words: dentin sensitivity, tooth erosion, anorexia nervosa, motivation, temporary dental restoration

Received: October 06, 2017; Accepted: October 24, 2017; Published: October 26, 2017 